\title{
Serum ICAM-1, VCAM-1 and E-selectin levels in patients with primary and secondary Sjögren's syndrome
}

\author{
Katarzyna J. Błochowiak, A-D, F, Anna Olewicz-Gawlik2, A, B, E, F, Dorota Trzybulska, ${ }^{2, C-F}$, Michalina Nowak-Gabryel, B, E, F, \\ Jarosław Kocięcki ${ }^{3, E, F}$, Henryk Witmanowski ${ }^{4}, \mathrm{E}, \mathrm{F}$, Jerzy Sokalski ${ }^{1, A, E, F}$ \\ 'Department of Oral Surgery, Poznan University of Medical Sciences, Poland \\ 2Department of Rheumatology and Clinical Immunology, Poznan University of Medical Sciences, Poland \\ ${ }^{3}$ Department of Ophthalmology, Poznan University of Medical Sciences, Poland \\ ${ }^{4}$ Department of Plastic, Reconstructive and Aesthetic Surgery, Nicolaus Copernicus University Torun, Collegium Medicum, Bydgoszcz, Poland \\ A - research concept and design; B - collection and/or assembly of data; $\mathrm{C}$ - data analysis and interpretation; \\ $D$ - writing the article; $E$ - critical revision of the article; $F$ - final approval of article
}

Address for correspondence

Katarzyna Błochowiak

E-mail: kasia@naszdentysta.com.pl

Funding sources

This study was supported by grant no.

502-14-02212331-09591, Poznan University

of Medical Sciences.

Conflict of interest

None declared

Acknowledgements

None declared

Received on September 21, 2015

Revised on November 16, 2015

Accepted on January 19, 2016

\begin{abstract}
Background. Typical features of Sjögren's syndrome (SS) are severe xerostomia and xerophthalmia which are basic diagnostic criteria.

Objectives. The aim of this study was to compare the serum levels of soluble (s) intercellular adhesion molecule 1 (sICAM-1), vascular cell adhesion molecule 1 (SVCAM-1) and sE-selectin between primary (pSS), secondary (sSS) and healthy subjects (HS). We correlated these results with selected clinical parameters of disease activity and parameters of the severity of xerostomia and xerophthalmia.
\end{abstract}

Material and methods. The serum levels of SICAM-1, SVCAM-1 and sE-selectin were determined by enzyme-linked immunosorbent assay (ELISA) in 16 patients with pSS, 18 with sSS and 15 HS. Eye dryness and xerostomia were assessed by the Schirmer's test, the Fox test and the visual analogue scale (VAS).

Results. The levels of SICAM-1 in pSS and SVCAM-1 in sSS patients were significantly higher when compared to HS ( $p=0.02$ and $p=0.048$, respectively). There were no differences between pSS and sSS. In pSS, sVCAM-1 correlated positively with VAS ( $r S=0.52, p=0.04$ ) and the Fox test ( $r S=0.66, p=0.01$ ). In $s S S$, sE-selectin correlated positively with sICAM-1 ( $r S=0.54, p=0.01)$, the duration of the disease $(r S=0.51$, $p=0.03)$ and negatively with the $S$ chirmer's test $(r S=0.59, p=0.04)$. slCAM-1 correlated positively with the erythrocyte sedimentation rate (ESR) value $(r S=0.59, p=0.01)$.

Conclusions. SVCAM-1 reflects xerostomia in pSS. sICAM-1 and SE-selectin may be additional parameters of SSS activity.

Key words: Sjögren's syndrome, xerostomia, IICAM-1, sE-selectin, sVCAM-1

DOI

10.17219/acem/61434

Copyright

Copyright by Author(s)

This is an article distributed under the terms of the

Creative Commons Attribution Non-Commercial License

(http://creativecommons.org/licenses/by-nc-nd/4.0/) 


\section{Introduction}

Recently, the importance of angiogenesis in the pathogenesis of autoimmune diseases related to chronic inflammation such as rheumatoid arthritis (RA), systemic lupus erythematosus (SLE) and Sjögren's syndrome (SS) has been highlighted., ${ }^{1,2}$ Observations from many studies support the existence of an important link between inflammation and angiogenesis in the pathogenesis of SS. ${ }^{1,2}$ In the labial salivary glands of SS patients, immunocytochemical studies have shown an increased expression of ICAM-1 and Eselectin on endothelial cells and of ICAM-1 on the epithelium. ${ }^{2,3}$ The expression and function of ICAM-1, VCAM-1 and E-selectin have been implicated in the pathogenesis of connective tissue diseases (CTD) by the interactions of circulating immune cells with vascular endothelium during extravasation and the interactions between $\mathrm{T}$ cells and antigen-presenting cells (APC). ${ }^{4}$

Typical features of SS are severe xerostomia and xerophthalmia, which are basic SS diagnostic criteria. The presence of both dry mouth and dry eyes classified patients with $93 \%$ sensitivity and $97.7 \%$ specificity. ${ }^{5}$ Decreased salivation markedly affects oral health and very often inhibits normal functioning. To determine xerostomia objectively, sialometry is applied to measure the quantity of resting and stimulated saliva secretion over the controlled time. Subjective tests which evaluate the real experience of xerostomia and the discomfort are of similar importance. The VAS and Fox test are carried out for this purpose.

The levels of soluble (s) ICAM-1, sVCAM-1 and sE-selectin in patients with pSS and sSS have not yet been compared. Most of the studies conducted so far have focused on the local expression of ICAM-1, VCAM-1 and E-selectin in salivary glands. Although elevated serum levels have been noticed, our concern is the possibility to correlate their values with basic inflammatory parameters such as the erythrocyte sedimentation rate (ESR) and other selected clinical findings. It seems important to determine how these levels affect the whole body. This would make it possible to use sICAM-1, sVCAM-1 and sE-selectin levels as additional, supporting parameters in the diagnosis and determination of the disease and concomitant inflammation severity.

Thus, the primary aim of this study was to measure and compare the serum levels of sICAM-1, sVCAM-1 and sEselectin in pSS, sSS and HS groups and to explore possible correlations between the concentrations of the selected molecules and the laboratory and clinical parameters of SS activity, including the severity of xerostomia and xerophthalmia.

\section{Material and methods}

\section{Study groups}

The study was comprised of 34 women with SS (16 with pSS and 18 with sSS), fulfilling the 2002 American-Eu- ropean Consensus Group classification criteria. In our study, the diagnosis of pSS required 4 of 6 criteria, including antibodies to SSA/SSB. A diagnosis of sSS has not yet been addressed by the American-European Consensus Group. In practice, we required the patients to fulfill the criteria for pSS and, additionally, the American College of Rheumatology criteria for an established CTD such as RA, SLE or mixed CTD (MCTD).$^{6-9}$ The patients were recruited consecutively in 2013 from the Department of Rheumatology and Clinical Immunology at Poznan University of Medical Sciences, Poland. Exclusion criteria included: previous radiotherapy to the head and neck, lymphoma, sarcoidosis, graft-versus-host disease, infections of the hepatitis $\mathrm{C}$ virus, human T-lymphotropic virus type I and HIV. A routine patient history was taken and physical and dental examinations were performed in each subject. Laboratory assessments included routine measurements of ESR (Westergren) and detection of antinuclear antibodies (ANA) by indirect immunofluorescence on Human Epithelial (HEp)-20-10 cells (Euroimmun, Lubeck, Germany) and their differentiation using ANA Profile3 (antibodies against nRNP/Sm, Sm, SS-A, Ro-52, SS-B, Scl-70, PM-Scl, Jo-1, centromere protein B, PCNA, dsDNA, nucleosomes, histones, ribosomal P-proteins and AMA-M2), (Euroimmun, Lubeck, Germany). Xerostomia (assessed by patients) was measured using the VAS and Fox test. To assess ocular sicca symptoms, the Schirmer's test was carried out. Fifteen age- and gender-matched healthy donors from the Regional Center of Blood Donation and Blood Treatment in Poznan formed the control group (healthy subjects, HS). The protocol for this study was approved by the Bioethics Committee of Poznan University of Medical Sciences, Poland (number $211 / 2013$ ). This study was performed in accordance with the ethical standards laid down in an appropriate version of the World Medical Association Declaration of Helsinki. Written informed consent was obtained from every subject before any study procedure was carried out.

\section{Sample collection}

Peripheral blood samples were collected from the antecubital vein in BD Vacutainer Rapid Serum Tubes (Becton, Dickinson and Company, Franklin Lakes, USA). After $5 \mathrm{~min}$, clots were removed by centrifugation at $4000 \mathrm{rpm}$ for $15 \mathrm{~min}$ at room temperature. The sera were stored at $-70^{\circ} \mathrm{C}$.

\section{ELISA}

sICAM-1, sVCAM-1 and sE-selectin levels in serum were determined by commercially available ELISA kits (R\&D Systems, Minneapolis, USA) for sICAM-1/CD54 (with a mean minimum detectable dose (MDD) of $0.096 \mathrm{ng} / \mathrm{mL}$ ), for sVCAM-1/CD106 (with a mean MDD of $0.6 \mathrm{ng} / \mathrm{mL}$ ) and for sE-selectin/CD62E (with a mean 
MDD of $0.009 \mathrm{ng} / \mathrm{mL}$ ). Absorbance was measured with an ELISA plate reader ELx800 (Bio-Tek Instruments, Winooski, USA) using KCjunior 1.11 analysis software (Bio-Tek, Winooski, USA).

\section{Assessment of xerostomia by Fox test and VAS}

Each subject completed a specially prepared questionnaire concerning afflictions, with special attention paid to the presence of oral dryness symptoms. The questionnaire consists of 10 items, 4 of which (1-4) show a significant correlation with reduced salivary flow. These questions concern a feeling of too little saliva in the oral cavity, a sense of oral dryness (xerostomia) while eating, difficulty in swallowing and the need to wash down dry food with water. Items in the Fox test:

1. Do you need to sip liquids to aid in swallowing dry foods?

2. Does your mouth feel dry when eating a meal?

3. Do you have difficulty swallowing any foods?

4. Does the amount of saliva in your mouth seem to be too little, too much, or you do not notice it?

5. Does your mouth feel dry at night or on awakening?

6. Does your mouth feel dry during the daytime?

7. Do you keep a glass of water by your bed?

8. Do you chew gum daily to relieve oral dryness?

9. Do you use hard candies or mints daily to relieve oral dryness?

10. Do you feel the need for frequent moistening of the oral cavity?

The Fox test score is measured as a proportion of positive to negative answers (yes/no) and expressed as a percentage value. $^{10,11}$

Assessment of the patient's xerostomia on the VAS consisted in the patient marking the severity of dry mouth on a $100 \mathrm{~mm}$ line. The VAS is a horizontal line, $100 \mathrm{~mm}$ in length, anchored by word descriptors. The VAS score is determined by measuring in $\mathrm{mm}$ from the left-hand end of the line to the point of the patient's mark. ${ }^{12,13}$

\section{Assessment of dry eye by Schirmer's test}

In 23 patients ( 11 with pSS and 12 with sSS), the Schirmer's test was carried out to assess the severity of dry eye. Both eyes were tested at the same time. Special paper strips were placed under the lower eyelid of each eye. The patients kept their eyes closed for $5 \mathrm{~min}$. Mechanical irritation resulted in the production of tears. After $5 \mathrm{~min}$, the paper was removed and measured to check how moist it was. More than $10 \mathrm{~mm}$ of moisture on the filter paper after $5 \mathrm{~min}$ is a sign of normal tear production. Both eyes normally release the same amount of tears. A score between of $6-10 \mathrm{~mm}$ is a sign of mild to moderate dry eye. Less than $6 \mathrm{~mm}$ of moisture on the filter paper after
5 min is a sign of severe dry eye. To report the severity of dry eye we used the average of both eyes.

\section{Statistical analyses}

The calculations were carried out with Microsoft Excel 2010 and STATISTICA v. 10 software (StatSoft Inc., Tulsa, USA). The distributions obtained at each step of data processing were evaluated for normality using the Shapiro-Wilk test. Depending on the number of groups analyzed, the differences between them were tested using the Mann-Whitney U test or the Kruskal-Wallis one-way analysis of variance (ANOVA) by ranks followed by post hoc multiple comparisons of the mean ranks. The Spearman's rank correlation analysis was used to find the associations between the levels of selected cytokines and other laboratory and clinical parameters of SS activity. Unless otherwise stated, the data are expressed as medians with interquartile ranges (IQR). The differences were considered to be statistically significant at $\mathrm{p}<0.05$.

\section{Results}

\section{Demography}

All the subjects were of Caucasian origin. Tables 1 and 2 present a demographic, laboratory and clinical profile of the SS patients.

\section{Serum sICAM-1, sVCAM-1 and sE-selectin levels}

Serum sICAM-1 levels in pSS patients were significantly higher than those of HS ( $p=0.02)$. Serum sVCAM-1 levels in sSS were significantly higher than those of HS $(\mathrm{p}=0.048)$. Concentrations of $\mathrm{sE}$-selectin in all studied groups were at a similar level. The detailed results are presented in Table 3. In pSS, the serum levels of sVCAM-1 correlated positively with VAS $\left(\mathrm{r}_{\mathrm{S}}=0.52, \mathrm{p}=0.04\right)$, as well as with the scores on the Fox test $\left(r_{S}=0.66, p=0.01\right.$ ) (Fig. $1 \mathrm{~A}$ and $1 \mathrm{~B}$, respectively). The levels of sE-selectin correlated positively with the levels of sICAM-1 $\left(r_{S}=0.54\right.$, $\mathrm{p}=0.01$ ) (Fig. 2A) and with the duration of the disease $\left(r_{S}=0.51, p=0.03\right)$ (Fig. $\left.2 B\right)$. The levels of sE-selectin correlated negatively with the Schirmer's test $\left(r_{S}=-0.59\right.$, $\mathrm{p}=0.04$ ) (Fig. 2C). The levels of sICAM-1 positively correlated with the ESR value $\left(\mathrm{r}_{\mathrm{S}}=0.59, \mathrm{p}=0.01\right.$ ) (Fig. 2D). No other significant correlations were found between the levels of molecules and the clinical parameters studied.

\section{Discussion}

Adhesive molecules such as ICAM-1, VCAM-1 and E-selectin contribute to the pathogenesis of SS in many 
Table 1. Characteristics of SS patients

\begin{tabular}{|c|c|c|c|}
\hline SS patients $(n=34)$ & pSS $(n=16)$ & sSS $(n=18)$ & HS $(n=15)$ \\
\hline Age (years) & $41.5(28.5)$ & $56.0(21.0)$ & $49(14)$ \\
\hline Gender (women/men) & $16 / 0$ & $18 / 0$ & $14 / 1$ \\
\hline BMl & $24.65(7.60)$ & $23.55(5.30)$ & \\
\hline Disease duration (years) & $4.5(4)$ & $3(9)$ & \\
\hline \multicolumn{4}{|c|}{ Connective tissue disease, $\mathrm{n}(\%)$} \\
\hline RA & - & $7(38.89)$ & \\
\hline SLE & - & $4(22.20)$ & \\
\hline MCTD & - & $1(5.55)$ & \\
\hline Others & - & $6(33.33)$ & \\
\hline \multicolumn{4}{|c|}{ Serological and clinical parameters of SS activity } \\
\hline Westergren ESR (mm/h) & $23.5(31)$ & $19(15)$ & \\
\hline ANA, n (\%) & $11(68.75)$ & $15(83.33)$ & \\
\hline Anti-SAA antibodies & SSA $10(62.50)$ & SSA $10(55.55)$ & \\
\hline Anti-SSB antibodies & SSB 7 (43.75) & SSB 4 (22.22) & \\
\hline $\begin{array}{l}\text { Other identified } \\
\text { ANA profile 3, n (\%) }\end{array}$ & Ro-52 $10(62.50)$ & $\begin{array}{c}\text { Ro-52 } 11 \text { (61.11) } \\
\text { dsDNA } 316.67 \\
\text { Sm } 1 \text { (5.55) } \\
\text { PCNA 1 (5.55) ribosomal-P-protein 3 } 16.67 \\
\text { centromeres B 1 (5.55) } \\
\text { PM-Scl } 1 \text { (5.55) } \\
\text { histones } 1 \text { (5.55) } \\
\text { nucleosomes } 1 \text { (5.55) } \\
\text { RNP 1 (5.55) }\end{array}$ & \\
\hline \multicolumn{4}{|c|}{ Organ involvement, n (\%) } \\
\hline Arthritis & $11(68.75)$ & $11(61.11)$ & \\
\hline Cutaneous & $4(25.00)$ & $3(16.17)$ & \\
\hline Peripheral nervous system & $5(31.25)$ & $3(16.67)$ & \\
\hline Pulmonary & $4(25.00)$ & $1(5.55)$ & \\
\hline Lymphadenopathy & $3(18.75)$ & $2(11.11)$ & \\
\hline Glandular & $1(6.25)$ & $1(5.55)$ & \\
\hline \multicolumn{4}{|c|}{ Current treatment, n (\%) } \\
\hline MTX & 0 & $5(27.78)$ & \\
\hline NSAID & $4(25.00)$ & $4(22.22)$ & \\
\hline Methylprednisolone & $4(25.00)$ & $6(33.33)$ & \\
\hline
\end{tabular}

BMI - body mass index; ESR - erythrocyte sedimentation rate; na - not applicable; RA - rheumatoid arthritis; SLE - systemic lupus erythematosus; MCTD - mixed connective tissue disease; ANA - antinuclear antibodies; MTX - methotrexate; NSAID - non-steroidal anti-inflammatory drugs; unless otherwise stated, the data are expressed as medians (interquartile range). 
aspects. In our study, the serum levels of sICAM-1 were elevated in pSS and the serum levels of sVCAM-1 were elevated in sSS as compared with HS. Similar results were obtained by Kapsogeorgou et al. They detected higher spontaneous expression of ICAM-1 in cell lines obtained from SS patients compared to the controls. ${ }^{3}$ The epithe-

Table 2. Oral and ocular characteristics of SS patients

\begin{tabular}{|c|c|c|}
\hline SS patients $(n=34)$ & pSS $(n=16)$ & $\mathrm{sSS}(\mathrm{n}=18)$ \\
\hline Xerostomia & $9(56.25)$ & $12(66.67)$ \\
\hline Dysphagia & $4(25.0)$ & $6(33.33)$ \\
\hline PtXer-VAS (mm) & $46.5(52.5)$ & $28.0(47.0)$ \\
\hline Fox test score (\%) & $45(40)$ & $55(30)$ \\
\hline \multicolumn{3}{|c|}{ Oral symptoms, n (\%) } \\
\hline Cheilitis simplex & $4(25.00)$ & $4(22.22)$ \\
\hline Aphthae & $2(12.5)$ & $1(5.55)$ \\
\hline Cheilitis angularis & $1(6.25)$ & $2(11.11)$ \\
\hline Tongue inflammation & $1(6.25)$ & $2(11.11)$ \\
\hline Stomatitis & $1(6.25)$ & $1(5.55)$ \\
\hline Cheilitis exfoliativa & $1(6.25)$ & 0 \\
\hline Oral paleness & $1(6.25)$ & 0 \\
\hline $\begin{array}{l}\text { Non-specific } \\
\text { ulcerations }\end{array}$ & 0 & $1(5.55)$ \\
\hline \multicolumn{3}{|c|}{ Ocular symptoms } \\
\hline $\begin{array}{l}\text { Schirmer's test }(\mathrm{mm}) \\
(\mathrm{pSS} n=11, \mathrm{sSS} n=12)\end{array}$ & $4(8)$ & $10.75(12)$ \\
\hline $\begin{array}{l}\text { Dryness of the eyes } \\
\text { (subjective assessment), } \\
n(\%)\end{array}$ & $11(68.75)$ & $13(72.22)$ \\
\hline
\end{tabular}

PtXer-VAS - patient xerostomia assessment on visual analogue scale; unless otherwise stated, the data are expressed as median (interquartile range). lial cells are the main target in the pathogenesis of SS and the main site of infiltration formation, which lead to a deterioration in the functioning of the salivary glands and other organs. ${ }^{5,14}$ Epithelial cells adjacent to the sites of intense inflammation and lymphocytic infiltrations have been shown to express high levels of ICAM-1, VCAM and E-selectin, which influence the interaction between epithelial cells and immunologic cells. The epithelial cells in SS are activated and act as non-professional APC. ICAM-1 and VCAM-1 are expressed on APC and binding lymphocyte function-associated antigen-1 (LFA-1), and the very late activation antigen-1 (VLA-1) receptors on T cells ensure stabilization of the APC/T cell synapsis. ${ }^{5}$ The possible consequence of this interaction is an augmentation of the inflammatory response either by directly activated $\mathrm{T}$ cells or by enhancing co-stimulatory and adhesion molecules such as ICAM-1 on the APC. ${ }^{5}$ This means that the activation of the epithelial component of salivary glands and other organs could be responsible for the increased levels of ICAM-1 and VCAM-1 in pSS and sSS patients, respectively, and for the positive correlations between the levels of ICAM-1 and the severity of inflammation in sSS patients. SS affects many organs and the similar mechanism of activation of the epithelial cells takes place not only in the salivary and lacrimal glands but in other organs as well, and is responsible for the elevated serum levels of ICAM-1 and VCAM- 1 . $^{8}$

Furthermore, the presence of proinflammatory cytokines such as interleukin (IL) $1 \beta$, tumor necrosis factor alpha (TNF- $\alpha$ ), IL-4 and interferon gamma (IFN- $\gamma$ ) in the walls of blood vessels adjacent to the sites of inflammatory infiltrations results in the expression of ICAM-1, VCAM-1 and E-selectin on endothelial cells and on leukocytes. ${ }^{1,4,14-16}$ TNF- $\alpha$ and IL-1 $\beta$ also induce de novo VCAM-1 expression. ${ }^{4}$ For a comprehensive view of SS, the determination of adhesive molecule-cytokine interactions is necessary. Injuries to the endothelium and its subsequent apoptosis in salivary glands significantly contribute to the pathogenesis of SS. ${ }^{17}$ Endotoxins and proinflammatory cytokines released from injuries induce the surface expression of adhesive molecules on endothelial cells, which results in inflammatory destruction. ${ }^{18}$ This explains the overexpression of adhesive molecules

Table 3. Serum levels of sICAM-1, sVCAM-1 and sE-selectin in the studied groups

\begin{tabular}{|c|c|c|c|}
\hline & sICAM-1 (ng/mL) & sVCAM-1 (ng/mL) & sE-selectin (ng/mL) \\
\hline $\operatorname{pSS}(n=16)$ & $221.26(60.95)$ & $576.82(518.35)$ & $28.03(7.86)$ \\
\hline sSS $(n=18)$ & $216.00(67.66)$ & 599.35 (509.07) & 23.69 (23.07) \\
\hline $\mathrm{HS}(\mathrm{n}=15)$ & $168.48(61.76)$ & $376.44(194.12)$ & $18.94(16.27)$ \\
\hline p-value\# & 0.02 (pSS vs HS) & 0.048 (sSS vs HS) & ns \\
\hline
\end{tabular}

The results are expressed as median (interquartile range); \# Kruskal-Wallis one-way ANOVA by ranks followed by post hoc multiple comparisons of the mean ranks. 
Fig. 1. Correlations between SVCAM-1 and PtXer VAS (A) and the results of the Fox test (B) in patients with pSS. The strength of the correlations was determined using Spearman's rank correlation coefficient; $p<0.05$ was considered statistically significant
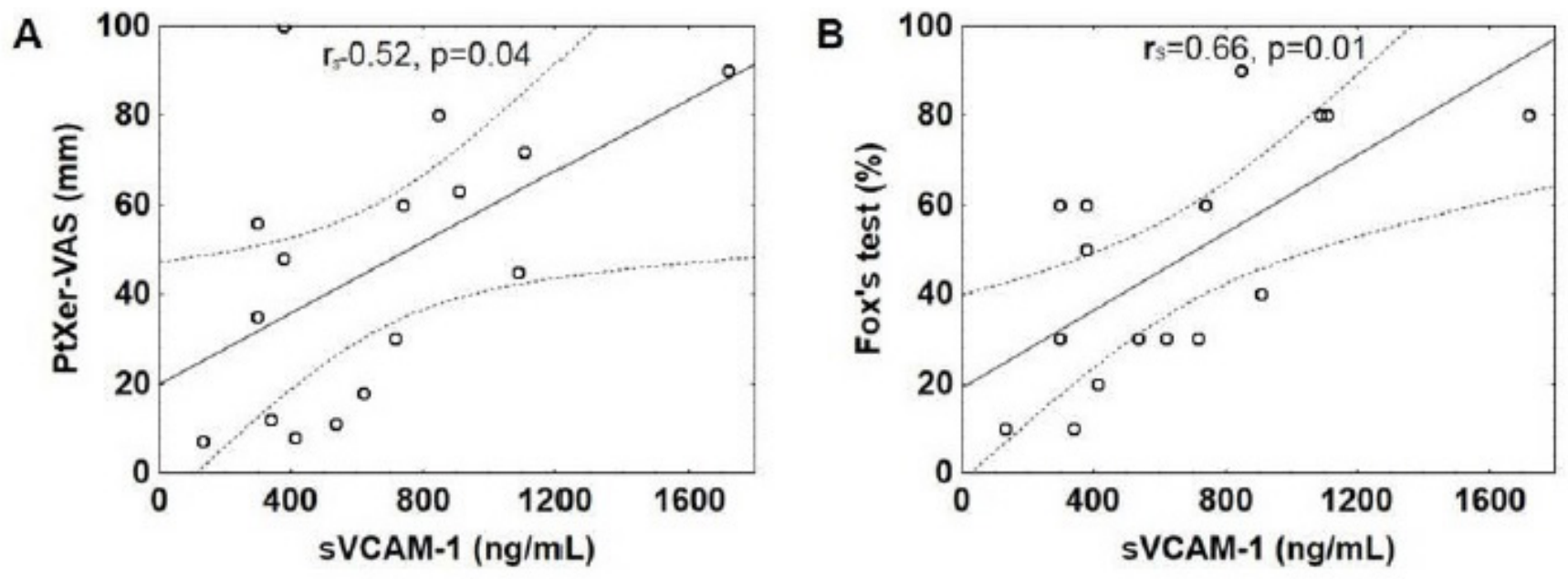

Fig. 2. Correlations between concentrations of sE-selectin and sICAM-1 (A), sSS duration (B) and the results of Schirmer's test (C) and between ESR and SICAM-1 levels (D) in patients with sSS. The strength of the correlations was determined using Spearman's rank correlation coefficient; $p<0.05$ was considered statistically significant
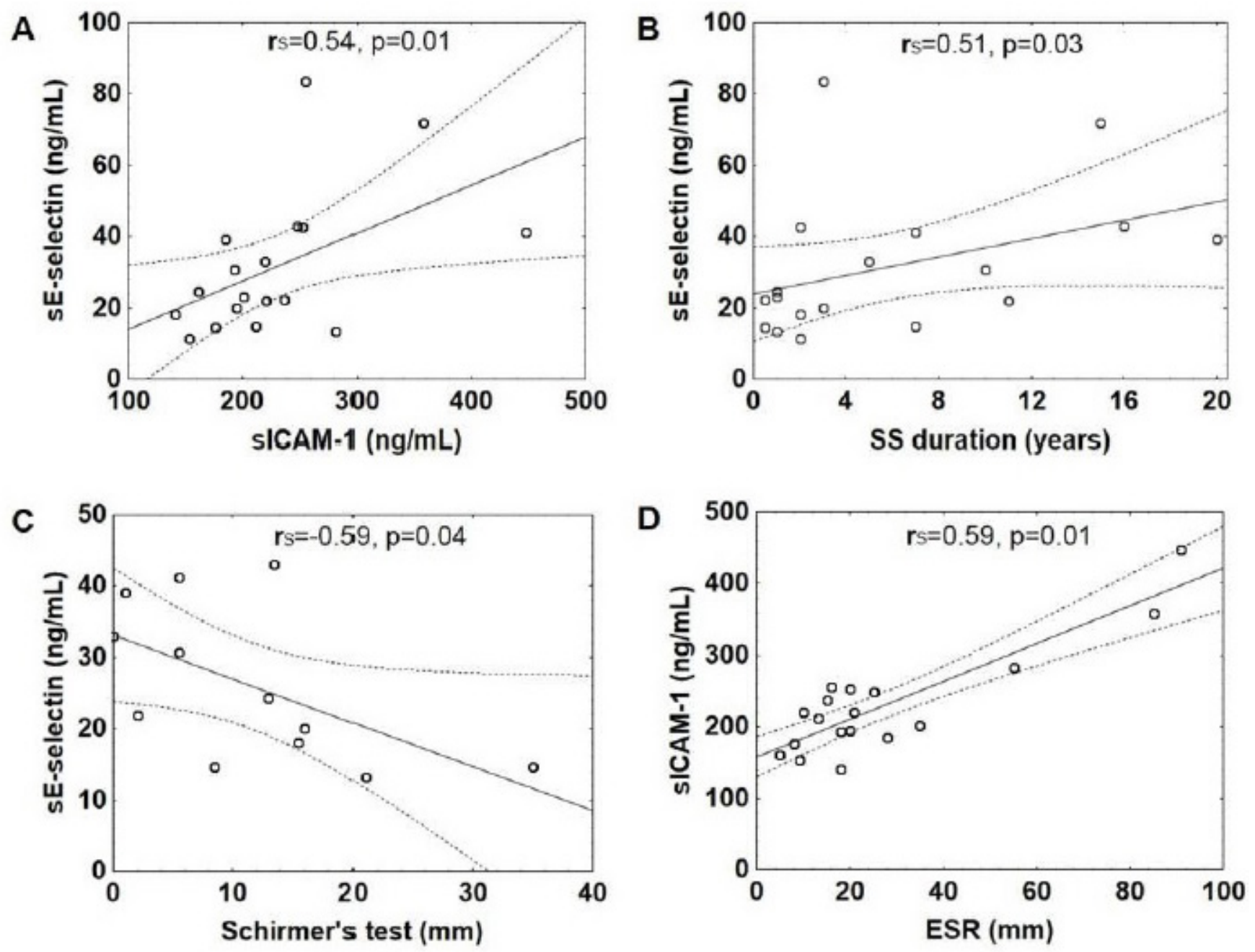
in damaged salivary glands in SS. ${ }^{18}$ Thus, the activation of epithelial cells and endothelial cells reflects the elevated serum levels of sICAM-1 and sVCAM-1 in pSS and sSS compared to HS.

In addition to being caused by aberrant angiogenesis and blood vessel injuries, the increased levels of adhesive molecules can be the result of the VEGF-A/VEGFR2 dependent mechanism of neovascularization. ${ }^{19}$ Elevated levels of VEGF appear in many CTDs. ${ }^{20}$ Additionally, VEGF promotes the migration of monocytes and lymphocytes into the extracellular matrix and sustains inflammation by endothelial cell expression of ICAM-1 and VCAM-1. 2,19,21 ICAM-1, VCAM-1 and E-selectin function as receptor molecules for circulating neutrophils. After binding, they reduce the blood flow of circulating cells and initiate their migration into the tissue. If the adhesion receptors are no longer needed, they are shed from the endothelial layer and circulate as soluble adhesion molecules. Therefore, the levels of circulating molecules are proportional to their previous activation level and blood vessel destruction. This can also explain the elevated serum levels of soluble adhesive molecules in pSS and sSS patients.

We did not find any differences between pSS and sSS in the levels of SICAM-1, sVCAM-1 and sE-selectin. It was found in recent studies comparing pSS and sSS that the intensity and frequency of some symptoms can vary. There is uncertainty whether sSS is merely a manifestation of the underlying disease or a true overlap of pSS with CTD. According to Salliot et al., subjects with sSS in the setting of SSc suffered from xerostomia, dry eye symptoms, the presence of anti-SSA/SSB antibodies and a classic histological pattern similar to those of subjects with pSS, again suggesting that these two entities are the same. ${ }^{22}$ The lack of clear results might arise from the different classification of sSS. The prevalence of sSS has been reported to range from $4 \%$ to $31 \%$, depending on the criteria applied, the methodological design and associated CTD. ${ }^{23}$ In our study, there were no differences in the levels of adhesive molecules in pSS and sSS patients. In our opinion, similar elevated serum levels of ICAM, VCAM and E-selectin can be a result of the similar histological pattern in pSS and sSS.

In our study, the levels of sVCAM-1 in pSS patients correlated with the severity of xerostomia assessed by VAS and the Fox test. In SS patients, the local environment of the inflamed gland leads to the dysfunction of the residual glandular units owing to the release of cytokines, metalloproteinases and autoantibodies. The earliest changes involve the development of small capillaries into high endothelial venules that secrete chemokines and express adhesive molecules, promoting the migration of immune cells into the glands. The infiltrating cells ( $\mathrm{T}$ and $\mathrm{B}$ cells, dendritic cells) interfere with glandular functions by means of the secretion of IL- 1 and TNF- $\alpha$, which inhibit the release of neurotransmitters such as acetylcholine, and the glandular response to these neurotransmitters ultimately leads to decreased saliva secretion. ${ }^{8}$ The blood supply of the salivary glands plays a role in salivation. In earlier studies, it was suggested that VCAM-1 in patients with SS may be related to vasculitis. ${ }^{4}$ These mechanisms could explain the correlation between the levels of sVCAM-1, reflecting salivary gland inflammation and glandular dysfunction, and the severity of xerostomia.

The levels of sICAM-1 positively correlated with sEselectin levels in sSS. This could suggest a similar contribution of these molecules in the pathomechanism of this disease, and their concentrations may be reflected by the activation of endothelial and epithelial cells. For a comprehensive view of sSS, these molecules should be determined together in material more representative for SS.

We also found a positive correlation between SE-selectin and sSS duration. Our result suggests that the higher concentration of this molecule may result from a longer activation of endothelium and epithelium in SS. Therefore, $\mathrm{sE}$-selectin may be considered a more representative parameter for SS progression.

In a study by Gao et al., it has been observed that ICAM-1 levels positively correlated with the progression of dry eye symptoms. ${ }^{24}$ We found only a significant correlation between the levels of $\mathrm{sE}$-selectin in $\mathrm{sSS}$ patients and the severity of dry eyes assessed by the Schirmer's test. Thus, this molecule seems to be the most representative parameter for the assessment of eye dryness in sSS.

The levels of sICAM-1 correlated positively with the ESR value in sSS patients. Its levels may reflect the previous activation of the epithelium and endothelium. Egerer et al. detected higher levels of ICAM-1 in pSS and SLE patients, as well as in sepsis, indicating a comparable stage of endothelial activation and therefore inflammation and subsequently vascular damage in these diseases. No differences were observed between patients with a localized infection and healthy controls regarding the levels of sE-selectin and sICAM-1. Interestingly, the endothelium in SLE and pSS appears to be chronically activated in contrast to the situation found in sepsis, where a high and constant level of ICAM-1 is usually detectable only for a period of days or weeks. ${ }^{25}$ The ICAM-1 level sustained at a constant and increased level can be a useful monitoring parameter of disease severity and the activity of inflammation. ${ }^{26}$

The limitations of this study arise mainly from the small sample size. Thus, it should be considered a pilot study. Our findings need to be verified in a larger SS population. In our study, a few patients were taking methylprednisolone, non-steroidal anti-inflammatory drugs (NSAIDs) and immunosuppressive drugs. There were no naïve patients with regard to both disease-modifying anti-rheumatic drugs and NSAIDs. These drugs may reduce the levels of proinflammatory cytokines and adhesive molecules. Moreover, the drug-cytokine interactions could be responsible for the disparity between the levels 
of adhesive molecules found in our study, especially in pSS and sSS patients. Additionally, many drugs applied in the therapy of hypertension, depression and other diseases can reduce salivation. Therefore, current and previous therapy and co-existing diseases and therapies might have interfered with our results but it is not clear whether these drugs can affect serum sICAM-1, sVCAM-1 and sEselectin levels in SS patients. In our study we correlated the levels of adhesive molecules with parameters of disease activity such as ESR. In our opinion, ESR is a good clinical marker for the assessment of a relationship between inflammation and disease activity in SS. Recently, two disease activity indices have been proposed: The European League Against Rheumatism (EULAR) Sjögren's syndrome patient reported index (ESSPRI) and the EULAR Sjögren's syndrome disease activity index (ESSDAI). ${ }^{27,28}$ These indices evaluate the effectiveness of therapy in pSS. In our clinical characteristics of SS patients, we included 6 of 12 domains (organ involvement) from the ESSDAI. Lack of a comprehensive evaluation of organ involvement according to the ESSDAI is a limitation of our study.

The limitations of the assessment of xerostomia and its relationship with the levels of adhesive molecules can arise from a subjective test of xerostomia. In our opinion, the Fox tests and VAS can be representative and helpful tests and they additionally describe the patient's discomfort in SS. Among the selected molecules, sVCAM-1 reflects xerostomia in pSS, and sICAM-1 and sE-selectin may be additional parameters of the activity of sSS. However, they cannot be applied for the differentiation of pSS and sSS.

\section{References}

1. Bodolay E, Koch AE, Kim J, Szegedi G, Szekanecz Z. Angiogenesis and chemokines in rheumatoid arthritis and other systemic inflammatory rheumatic diseases. J Cell Mol Med. 2002;6:357-376.

2. Lisi S, Sisto M, D'Amore M, Lofrumento DD. Emerging avenues linking inflammation, angiogenesis and Sjögren syndrome. Cytokine. 2013;61:693-703.

3. Kapsogeorgou EK, Dimitriou R, Abu-Helu F, Moutsopoulos M, Manonssakis MN. Activation of epithelial and myoepithelial cells in the salivary glands of patients with Sjögren's syndrome: High expression of intercellular adhesion molecule-1 (ICAM.1) in biopsy specimens and cultured cells. Clin Exp Immunol. 2001;124:126-133.

4. Turkcapar N, Sak SD, Saatci M, Duman M, Olmez U. Vasculitis and expression of vascular cell adhesion molecule-1, intercellular adhesion molecule-1, and e-selectin in salivary glands of patients with Sjögren's syndrome. J Rheumatol. 2005;32:1063-1070.

5. Mitsias DI, Kapsogeorgou EK, Moutsopoulos HM. Sjögren's syndrome:Whyautoimmuneepithelitis? OralDiseases.2006;12:523-532.

6. Vitali C, Bombardieri S, Jonsson R. Classification criteria for Sjögren syndrome: A revised version of the European criteria proposed by the American-European Consensus Group. Ann Rheum Dis. 2002;61:554-558.
7. Fox RI, Liu AY. Sjögren's syndrome in dermatology. Clinics in Dermatology. 2006;24:393-413.

8. Fox RI. Sjögren's syndrome. Lancet. 2005;366:321-331.

9. Carr AJ, Ng WF, Figueiredo F, Macleod RI, Greenwood M, Stainers K. Sjögren's syndrome-an update for dental practitioners. Br Dental J. 2012;213:353-357.

10. Malicka B, Kaczmarek U, Skośkiewicz-Malicka K. Prevalance of xerostomia and salivary flow rate in diabetic patients. Adv Clin Exp Med. 2014;23:225-233.

11. Wörnberg Gerdin E, Einarson S, Jonsson M, Aronsson K, Johansson I. Impact of dry mouth conditions on oral health-related quality of life in older people. Gerodontology. 2005;22:219-226.

12. Jamison RN, Gracely RH, Raymond SA, et al. Comparative study of electronic vs paper VAS ratings: A randomized, crossover trial using healthy volunteers. Pain. 2002;99:341-347.

13. Bolognese JA, Schnitzer TJ, Ehrich EW. Response relationship of VAS and Likert scales in osteoarthritis efficacy measurement. Osteoarthritis Cartilage. 2003;11:499-507.

14. Moriyama M, Hayashida JN, Toyoshima T, et al. Cytokine/chemokine profiles contribute to understanding the pathogenesis and diagnosis of primary Sjögren's syndrome. Clin Exp Immunol. 2012;169:17-26.

15. Roescher N, Tak PP, Illei GG. Cytokines in Sjögren's syndrome. Oral Diseases. 2009;15:519-526.

16. Steinfeld S, Simonart T. New approaches to the treatment of Sjögren's syndrome: Soon beyond symptomatic relief? Dermatology. 2003;207:6-9.

17. Voulgarelis M, Tzioufaz AG. Pathogenetic mechanisms in the initianion and perpetuation of Sjögren's syndrome. Nat Rev Rheumatol. 2010;9:529-537.

18. Gerli R, Vaudo G, Bocci EB, et al. Functional impairment of the arterial wall in primary Sjögren's syndrome: combined action of immunologic and inflammatory factors. Arthritis Care Res (Hoboken). 2010;62:712-718.

19. Sisto M, Lisi S, Ingravallo G, Lofrumento DD, D'Amore M, Ribatti $D$. Neovascularization is prominent in the chronic inflammatory lesions of Sjögren's syndrome. Int J Exp Path. 2014;95:131-137.

20. Carvalho JF, Blank M, Shoenfeld Y. Vascular endothelial growth factor (VEGF) in autoimmune diseases. J Clin Immunol. 2007;27:246-256.

21. Sisto M, Lisi S, Lofrumento DD, D'Amore M, Frassanito MA, Ribatti D. Sjögren's syndrome pathological neovascularization is regulated by VEGF-A stimulated TACE-dependent crosstalk between VEGFR2 and NF-KB. Genes Immun. 2012;13:411-420.

22. Salliot $C$, Mouthon $L$, Ardizzone $M$, et al. Sjögren's syndrome is associated with and not secondary to systemic sclerosis. Rheumatology. 2007;46:321-326.

23. Hernández-Molina G, Ávilo-Casado C, Cárdenas-Veiázquez F, et al. Similarities and differences between primary and secondary Sjögren's syndrome. J Reumathol. 2010;37:800-808.

24. Gao J, Morgan G, Tieu D, et al. ICAM-1 expression predisposes ocular tissues to immune-based inflammation in dry eye patients and Sjögrens syndrome-like MRL/Ipr mice. Exp Eye Res. 2004;78:823-835.

25. Egerer K, Feiste E, Rohr U, Pruss A, Burmester GR, Dörner T. Increased serum soluble CD14, ICAM-1 and E-selectin correlate with disease activity and prognosis in systemic lupus erythematosus. Lupus. 2000;9:614-621.

26. Bertorello R, Codone MP, Contini $P$, et al. Increased levels of interleukin-10 in saliva of Sjögren's syndrome patients: Correlation with disease activity. Clin Exp Med. 2004;4:148-151.

27. Seror R, Vitali C, Ravaud P, et al. EULAR Sjögren's syndrome disease activity index: Development of a consensus systemic disease activity index for primary Sjögren's syndrome. Ann Rheum Dis. 2010;69:1103-1109.

28. Seror $\mathrm{R}$, Theander $\mathrm{E}$, Bootsma $\mathrm{H}$, et al. Outcome measures for primary Sjögren's syndrome: A comprehensive review. J Autoimmun. 2014;51:51-56. 